\title{
Sedation reversal with flumazenil?
}

\author{
A randomised crossover trial of post-operative cognitive and psychomotor recovery from benzodiazepine sedation: \\ effects of reversal with flumazenil over a prolonged recovery period N. M. Girdler, K. J. Fairbrother, J. P. Lyne, N. \\ Neave, A. Scholey, N. Hargaden, K. A. Wesnes, J. Engler and N. A. Rotherham Br Dent J 2002; 192: 335-339
}

\section{Objective}

To study the post-operative cognitive and psychomotor recovery from midazolam conscious sedation, after reversal with the benzodiazepine antagonist flumazenil over a prolonged recovery period.

\section{Design}

A prospective, double-blind, randomised, crossover trial.

\section{Setting}

Out-patient Sedation Department, Newcastle Dental Hospital and School

\section{Method}

Eighteen patients, ASA I or II, received midazolam on two separate occasions to undergo equivalent dental treatment. Following treatment patients were reversed with intravenous flumazenil or saline (placebo) at alternate appointments. Assessment of mood and cognitive function was undertaken using a highly sensitive and specific computerised battery of cognitive tests administered by telephone. Cognitive and psychomotor tests were administered prior to sedation and every hour for 6 hours post reversal.

\section{Results}

Results indicated no significant effect of flumazenil on simple reaction time and choice reaction time but did show a trend of reversing the effects of midazolam on numeric working memory and word recognition.

\section{Conclusion}

The cognitive and psychomotor effects of the sedation were not fully reversed by flumazenil. Cognitive impairments were still present up to 6 hours post-reversal, despite patients appearing clinically more alert. This has important implications for treatment protocols and discharge instructions.

\section{IN BRIEF}

- Flumazenil does not completely reverse all aspects of conscious sedation at clinical doses.

- Patients who have been reversed should not be prematurely released into an unmonitored environment or discharged home early.

- Because of its shorter half-life, patients may show some residual sedation, after flumazenil administration

- Patients and their escorts still need full verbal and written postoperative instructions in the same manner as those patients who have not been reversed.

\section{COMMENT}

Midazolam has the been the principal drug used for intravenous sedation in dentistry in the UK for some years. Its use has an excellent track record for patient management and safety. One of the significant improvements in this respect has been the availability of the benzodiazepine antagonist flumazenil; its principle use being for the emergency reversal of benzodiazepine induced conscious sedation.

There have been a number of studies published regarding the effects of flumazenil on the psychomotor recovery of patients but most of these studies have only looked at the first couple of hours post-sedation. Much of the post-operative recovery of conscious sedation patients happens once they have left the surgical environment and therefore in an unmonitored environment at home. This recovery may be prolonged and it would be useful to know how flumazenil affects patients over a longer period of time. This study attempts to do that.

Eighteen patients were treated on two separate occasions each two weeks apart with the same dose of intravenous midazolam for restorative dentistry. After 45 minutes they were injected with either normal saline or flumazenil and they then completed a series of cognitive and psychomotive tests every hour for six hours. The first two of these were done in the recovery area and the next four were done at home by a novel touch-tone phone system. The study looked at mood, attention and memory, reaction times and number and word memory.

The results show that flumazenil does not appear to reverse the effects of midazolam sedation in terms of simple reaction time or in choice reaction time. The results therefore suggest that flumazonal is not effective in reversing the full cognitive impairment of midazolam use. At the end of the test period the reaction times are no different from those seen when midazolam sedation is not reversed and patients will therefore still potentially have problems with cognitive tasks. The authors report however that when patients self report their alertness, they feel that they have re-gained their pre-sedation scores. These findings obviously negate the use of subjective assessment for assessing patients for discharge.

This paper adds significantly to the evidence base regarding the use of flumazenil for reversal of intravenous conscious sedation with benzodiazepines and reinforces the existing practice that patients reversed with flumazenil should not be discharged early and should receive the same post-operative care and instructions as those who do not have their sedation reversed.

Jason Leitch

Clinical Lecturer, Oral Surgery and Sedation

Glasgow Dental Hospital and School 